\title{
A randomized, controlled, parallel group, multicenter, pivotal study to assess the safety and effectiveness of the epidural space verification with the CompuFlo ${ }^{\circledR}$ Epidural Computer Controlled System (CompuFlo)
}

\author{
D. Dobecki, R.E. Gebhard, T. Muller-Bertram, M.Walker, J.Shi, S.llic \\ San Diego Pain Institute, San Diego, CA USA
}

\begin{abstract}
Background and Goal of Study: Successful and safe performance of epidural anesthesia or epidural injections relies on correct identification of the epidural space. While methods for simple and objective identification of the ES have been proposed, most anesthesiologists and/or pain physicians still utilize either the subjective manual feeling of a loss of resistance (LOR) or objective but relatively invasive radiological confirmation via fluoroscopy (1). Primary objective of the study was to determine whether the success rate of performance of lumbar epidural anesthesia with the CompuFlo to identify the epidural space is equivalent to to performance of lumbar epidural anesthesia with the loss of resistance technique (LOR) to identify the epidural space.
\end{abstract}

Materials and Methods: Study was designed by Scientific Advisory Board as prospective, randomized, open label and approved by the IRB/EC and the U.S. Food and Drug Administration (FDA), to assess the safety and effectiveness of the CompuFlo, pressure sensing technology in identification of the epidural space when compared to standard of medicine as part of the acute and chronic pain management. Patient randomization was stratified according to body mass index (BMI), success of the procedure was verified with the correct spread of dye demonstrated by fluoroscopy as determined by the independent observer and the patient outcome were assessed utilizing Patient's Global Impression of Change (PGIC)Scale (2).

Results: This is a preliminary report of 219 subjects enrolled in the study for whom epidural procedure was needed as part of the chronic pain management.

\begin{tabular}{|l|c|c|c|}
\hline N & $\begin{array}{c}\text { Standard } \\
\text { /LOR }\end{array}$ & Compuiflo & P value \\
\hline Median Age (IQR) - yr & 105 & 114 & \\
\hline Gender Male - no. (\%) & $59(50-66)$ & $56(47-64)$ & 0.34 \\
\hline Race White - no. (\%) & $93(42 \%)$ & $54(47 \%)$ & 0.50 \\
\hline BMI $\geq 31-$ no. (\%) & $73(70 \%)$ & $76(67 \%)$ & 0.67 \\
\hline Success - no. (\%) & $105(100 \%)$ & $114(100 \%)$ & 1.00 \\
\hline Median PGIC (IQR) & $5.0(4.0-$ & $4.5(2.8-$ & 0.11 \\
\hline
\end{tabular}
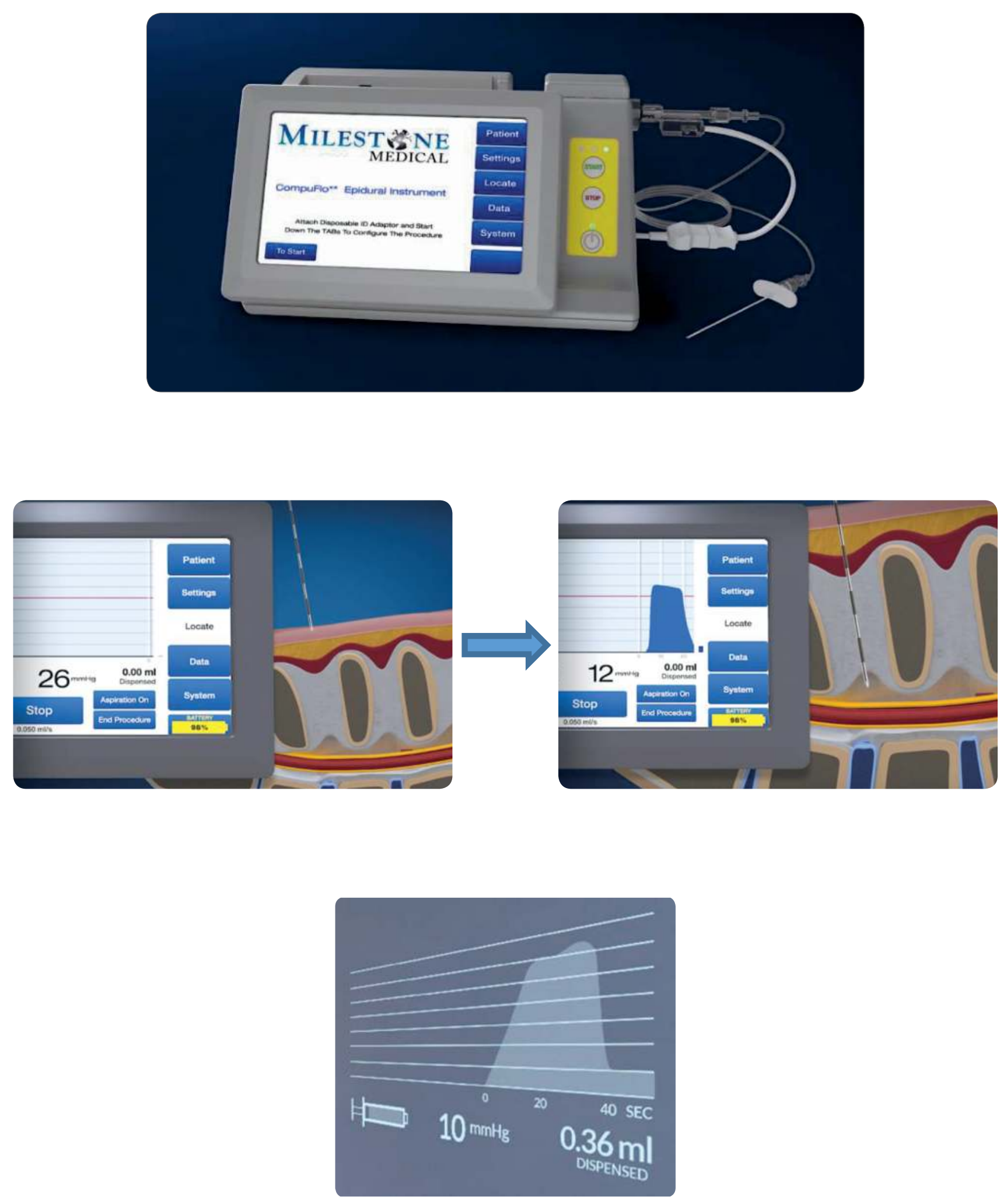

\section{CompuFlo ${ }^{\circledR}$ Audio /Visual Confirmation of the Epidural Space Location}

Discussion: This initial data suggest that identification of the epidural by utilizing a computerized injection pump technology and obtaining real-time pressure measurements from the needle tip, results in equivalent success rate, outcome and safety when compared to fluoroscopy.

Conclusion(s): Utilization of the CompuFlo technology may have the potential to avoid exposure of the patient to radiation without compromising procedure safety nor effectiveness.

References:

1. Hurst H, Bolton J.J Manipulative Physiol Ther. 2004 Jan; 27(1):26-35

2. Ghelber $O$ et al. Reg Anesth Pain Med 2008; 33:346-52 